\title{
Extracorporeal shockwave lithotripsy: first 1000 cases at the London Stone Clinic
}

\author{
G DAS, J DICK, M J BAILEY, M S FLETCHER, D R WEBB, M J KELLETT, \\ H N WHITFIELD, J E A WICKHAM
}

\begin{abstract}
One thousand patients underwent extracorporeal shockwave lithotripsy for renal and ureteric calculi at this clinic. An overall success rate of $91.8 \%$ was achieved (stone free or less than $2 \mathrm{~mm}$ fragments at three months) and for stones measuring $1 \mathrm{~cm} 96.3 \%$. Lithotripsy produced extremely low morbidity, and no deaths have occurred at the clinic. Patients who had lithotripsy alone had a mean hospital stay of three days and in most instances were able to perform their full range of activities on discharge. Planned combination of lithotripsy with minimally invasive endourological procedures such as percutaneous nephrolithotomy and ureterorenoscopy has allowed us to extend the range of treatable cases to include large stones. Prophylatic use of Double-J ureteric stents in selected cases has reduced the incidence of obstruction by stone fragments after lithotripsy, thereby decreasing morbidity and hospital stay.
\end{abstract}

\footnotetext{
London Stone Clinic and Academic Unit, Institute of Urology, London WC2H 8JE

G DAS, MS, FRCSED, lecturer in urology

J DICK, MRCP, FRCS, senior registrar, St Peter's Hospitals

M J BAILEY, MS, FRCS, senior registrar, St Peter's Hospitals

M S FLETCHER, MS, FRCS, assistant urologist, London Stone Clinic

D R WEBB, FRACS, assistant urologist, London Stone Clinic

M J KELLETT, MB, FRCR, consultant radiologist, St Peter's Hospitals

H N WHITFIELD, MCHIR, FRCS, consultant urologist, St Bartholomew's Hospital

J E A WICKHAM, MS, FRCs, director, academic unit, Institute of Urology

Correspondence to: Mr G Das, Academic Unit, Institute of Urology, London WC2H 8JE.
}

\section{Introduction}

The first lithotripter in the United Kingdom was installed at this clinic in November 1984. By October 1986, 1000 unselected patients had undergone extracorporeal shockwave lithotripsy for renal and ureteric calculi at this centre. A further 600 patients have also been treated by percutaneous nephrolithotomy or ureterorenoscopy.

The Dornier lithotripter produces fragmentation of renal calculi by extracorporeally administered shock waves. Patients are anaesthetised and positioned in a water bath on a hydraulic cradle. The calculi are accurately located by biplanar fluoroscopy, which also monitors the progress of fragmentation. Shock waves are generated by a high voltage underwater spark discharge and are focused on to the calculus by an ellipsoid reflector. The acoustic properties of the human body are similar to those of water, allowing the shock waves to pass through soft tissues with little attenuation until they reach a fluid solid interface, in this case, the stone surface. Here the shockwave energy is liberated as a mixture of stress and shear forces, resulting in fragmentation of the calculus. Lithotripters have been in clinical use in Germany since 1980, and over 120 machines are in use world wide. The safety of the procedure was initially evaluated by animal experiments and has been clinically confirmed by the successful treatment of over 300000 patients world wide.

\section{Patients and methods \\ REFERRAL AND INVESTIGATIONS}

Patients were referred by urologists, nephrologists, and general surgeons. Few were referred directly by their general practitioner. The suitability of a patient for extracorporeal shockwave lithotripsy was assessed by a reviewing panel of urologists and radiologists. Satisfactory drainage of the upper tracts is essential for good results, as compromised drainage prevents passage of stone fragments and causes obstruction. Patients whose calculi were 
unsuitable for lithotripsy alone were offered other treatments (available at the same centre), such as percutaneous extraction for calculi in narrow. necked calices and bulky staghorn stones and ureteroscopic retrieval for lower ureteric stones. Lithotripsy was also unsuitable for patients with an abnormal clotting profile, although it was possible to treat some with this problem after their clotting disorder had been medically corrected. Those whose body weight was over $135 \mathrm{~kg}$, who would overload the hydraulic cradle of the Dornier machine, and children under 3 years of age, who would not fit on the cradle, were not treated with lithotripsy.

Patients were admitted the day before treatment. A thorough clinical examination was peformed and blood sent to the laboratory for full blood count, biochemical profile, hepatitis antigen tests, and clotting screen. A midstream specimen of urine was obtained for culture and routine analysis. Patients were given trimethoprim $200 \mathrm{mg}$ twice a day by mouth as a prophylactic measure.

On the morning of treatment, plain abdominal $x$ ray films were taken to confirm the presence and site of the calculi. An ultrasound scan of the kidneys was performed to exclude or confirm a dilated pelvicaliceal system and as a baseline parameter for comparison in the event of postoperative obstruction.

\section{LITHOTRIPSY}

Most treatments were carried out under general anaesthesia, but a few patients were given an epidural anaesthetic. Renal calculi were fragmented by the Dornier lithotripter in the standard manner. ${ }^{1}$ Ureteric calculi situated above the pelvic brim were manipulated back into the renal pelvis by flushing with isotonic saline through a ureteric catheter and then treated (the "push-bang" technique) or fragmented in place if retrograde manipulation was unsuccessful. For large calculi, Double-J ureteric stents were inserted endoscopically under the same anaesthetic before lithotripsy as a prophylaxis against obstruction by lodged ureteric fragments after lithotripsy. After induction of anaesthesia an indwelling urethral catheter was inserted as lithotripsy produces temporary haematuria of about three hours' duration. To produce a brisk diuresis to facilitate passage of stone fragments $20 \mathrm{mg}$ of frusemide was given as an intravenous bolus towards the end of treatment.

\section{POSTOPERATIVE PERIOD AND FOLLOW UP}

The urethral catheter was removed when the haematuria cleared, and intravenous fluids were discontinued once the patients were drinking satisfactorily, usually the day after treatment. On day 2, plain radiography (KUB) was performed to confirm fragmentation and note clearance of fragments. Patients were allowed to go home if they had no symptoms or fever. They were instructed to complete their five day course of trimethoprim and report for first follow up in 10 days. A final follow up was requested at the end of three months.

\section{Results}

Altogether, 1000 patients underwent extracorporeal shockwave lithotripsy, 719 men $(71 \cdot 9 \%)$ and 281 women $(28 \cdot 1 \%)$. The mean age was $46 \cdot 3$ years (range 6-88 years). Planned combination of lithotripsy and percutaneous nephrolithotomy/ureteroscopy was performed in 89 patients (table I). Percutaneous nephrolithotomy followed by lithotripsy was performed for calculi greater than $3 \mathrm{~cm}$ : the stone mass was debulked by the first procedure and the remnant treated by lithotripsy. This treatment schedule was necessary as lithotripsy alone for large calculi produces a fragment mass greatly in excess of the ureter's capacity to clear fragments, resulting in obstruction. Lower ureteric calculi were removed by ureteroscopy. The push-bang technique as described above was used for ureteric calculi situated above the pelvic brim. The average size of stones treated by the push-bang method was $1.07 \mathrm{~cm}$.

To achieve good stone clearance repeat lithotripsy or subsequent percutaneous nephrolithotomy/ureteroscopy was performed in 114 cases (table II). Repeat lithotripsy or subsequent "toilet" percutaneous nephrolithotomy was performed to clear large pelvicaliceal fragments. Lower ureteric fragments causing obstruction were removed ureteroscopically. These auxiliary procedures were required in patients with large stone masses.

Success, as internationally agreed, was defined as stone free at three months or reduction into particles of less than $2 \mathrm{~mm}$, which would be expected to pass spontaneously..$^{36}$ By these criteria, $918(91 \cdot 8 \%)$ procedures were successful and $82(8 \cdot 2 \%)$ unsuccessful. Of the 918 successes, 642 patients were stone free, while 276 had asymptomatic $<2 \mathrm{~mm}$ fragments at last follow up. For stone size of $1 \mathrm{~cm}$ or less $96.3 \%$ procedures were successful, for stones $1-2 \mathrm{~cm} 92 \cdot 1 \%$ were successful, and for stones $>2 \mathrm{~cm}$ $84 \%$ were successful.

The mean hospital stay for patients who underwent lithotripsy only was three days, and for patients who underwent combined or auxiliary procedures this increased to 7.5 days. A mean of 1200 shocks were delivered at each procedure (range 100-4000).

\section{COMPLICATIONS RELATED TO EXTRACORPOREAL SHOCKWAVE LITHOTRIPSY}

No deaths occurred in this series or in any of the 1600 patients treated at the clinic to date. After lithotripsy obstruction by stone fragments in the ureter, the obstructing "steinstrasse" (stone street) was the commonest complication - $6 \cdot 8 \%$. Normally fragments move down the ureter, producing the characteristic $x$ ray appearance of a steinstrasse. Obstruction occurs when the collection of particles is held up in the ureter, showing clinically by loin pain and often complicated by superadded infection. Needle nephrostomy under local anaesthetic gave relief of obstruction in these cases. Postoperative fever of $>37.5^{\circ} \mathrm{C}$ for more than 24 hours occurred in $2.9 \%$ of all cases. Other complications included haemorrhage (three patients), cardiac arrhythmias, and appreciable cutaneous bruising at entry and exit sites of shock waves (15\%). All were treated as described in an earlier report. ${ }^{2}$

TABLE I-Procedures carried out on 1000 patients

\begin{tabular}{lc}
\hline Procedure. & No of patients \\
\hline Extracorporeal shockwave lithotripsy (ESWL) alone & 758 \\
Push-bang & 153 \\
ESWL+percutaneous nephrolithotomy & 66 \\
ESWL+ureteroscopy & 16 \\
Push-bang+ureteroscopy & 4 \\
Push-bang+ percutaneous nephrolithotomy & 2 \\
ESWL + percutaneous nephrolithotomy+ureteroscopy & 1 \\
\hline
\end{tabular}

TABLE II-Repeated procedures in 114 patients

\begin{tabular}{lc}
\hline Auxiliary procedure & No of patients \\
\hline Repeat extracorporeal shockwave lithotripsy (ESWL) & 32 \\
Repeat ESWL+percutaneous nephrolithotomy & 5 \\
Percutaneous nephrolithotomy & 46 \\
Percutaneous nephrolithotomy+ureteroscopy & 7 \\
Repeat ESWL+ureteroscopy & 2 \\
Ureteroscopy & 22 \\
\hline
\end{tabular}

\section{Discussion}

Several important points emerge from these results. Our overall success rate of $91.8 \%$ in all categories of stone size is similar to those of experienced continental and American centres. ${ }^{3478}$ Recently, the other British lithotripter centre at St Thomas's reported a much lower success rate of $44 \cdot 1 \%{ }^{9}$. It is notable that most of the patients at St Thomas's Hospital were referred from outside, many as day cases. As this was the only lithotripter available in the National Health Service, patients came from outside hospitals, were treated at St Thomas's Hospital, and returned to the referring hospital for management and follow up. In our clinic and other experienced centres patients are managed by the same team from preoperative assessment to discharge and follow up. The only patients treated as day cases at our centre were from St Bartholomew's and St Paul's, where the urologists are trained in shockwave lithotripsy and related problems. It is therefore not surprising that optimum results were obtained at centres where advanced endourological skill was available throughout the patient's clinical course.

In our series of 1000 patients, $751(75 \cdot 1 \%)$ completed full follow up, 642 of whom were stone free, $86 \mathrm{had}<2 \mathrm{~mm}$ residual fragments, and 23 had fragments $>2 \mathrm{~mm}$. Two hundred and forty nine $(24.9 \%)$ patients did not attend the three month consultation, 190 of whom had fragments $<2 \mathrm{~mm}$ and $59 \mathrm{had}>2 \mathrm{~mm}$ fragments. 
A total of 82 patients $(8 \cdot 2 \%)$ had residual fragments $>2 \mathrm{~mm}$ at their last follow up and were regarded as failures of treatment. Many would have (or indeed have) passed these fragments spontaneously in due course. Unfortunately, as indicated above, 59 were lost to follow up, highlighting the difficulties of ensuring a satisfactory follow up protocol in patients from overseas. The rest were offered repeat lithotripsy or percutaneous nephrolithotomy or both for upper tract fragments or ureteroscopic extraction for lower ureteric particles. In most of these cases further treatment was turned down by the patient.

Fragments of $<2 \mathrm{~mm}$ were present in 276 cases $(27 \cdot 6 \%)$. As indicated in our previous report gravel may persist in dependent calices with no clinical effect. ${ }^{6}$ The fate of these finely fragmented particles will need to be evaluated in the future. There is no evidence that lithotripsy is associated with a higher rate of recurrence than after open surgery or percutaneous nephrolithotomy. ${ }^{7}$ Moreover, repeat lithotripsy or percutaneous nephrolithotomy can be used to clear these kidneys at a later date if clinically indicated.

Four patients subsequently underwent open surgery. Two nephrectomies were performed, one for secondary haemorrhage after combination treatment by lithotripsy+percutaneous litholithotomy for a large calculus. Nephrectomy was carried out overseas where facilities for more conservative measures to arrest haemorrhage, such as selective renal arterial embolisation, were not available. The second nephrectomy was performed in a woman who developed pyelonephritis in a poorly functioning kidney which had had lithotripsy six months earlier for a recurrent staghorn calculus. Two open ureterolithotomies were performed, the first after failed ureteroscopic removal of lower ureteric fragments following lithotripsy to a $1 \mathrm{~cm}$ mid-ureteric calculus and the second to relieve obstruction in a patient with renal failure and a single kidney.

With experience we learnt the value of using Double-J stents. Earlier studies showed that the ureter dilates around a stent in response to its presence in the lumen. ${ }^{10} \mathrm{This}$ principle was exploited in treating stones of borderline size $(2-3 \mathrm{~cm})$-not large enough to warrant the separate procedure of percutaneous debulking yet big enough to produce fragment mass capable of creating an obstructing steinstrasse. In these cases the prophylatic use of Double-J stents has $(a)$ circumvented postoperative obstruction and all its clinicopathological sequelae of loin pain and septicaemia and $(b)$ helped in clearing fragments. Stents were left in place until satisfactory clearance had been obtained, and subsequent removal entailed a minor endoscopic procedure.

Thus our present treatment policy is as follows: for a stone mass $<2 \mathrm{~cm}$ lithotripsy alone; for $2-3 \mathrm{~cm}$ lithotripsy+prophylactic placement of Double-J stent; for $>3 \mathrm{~cm}$ percutaneous debulking followed by lithotripsy.

The practice of extracorporeal shockwave lithotripsy has under- gone refinements of technique and policy over the years. Comparing our earlier experience, there have been appreciable modifications. ${ }^{111}$ Two previous publications mention the mean number of shock waves delivered at each treatment as 779 and 944 respectively with a maximum of 2000 . This paper reports a mean number of shock waves of 1200 , and maximum shock waves 4000 , reflecting treatment of larger and denser stone masses. The incidence of auxiliary intervention was $8 \%$ in our first 50 patients, and we commented that this would be expected to rise as more difficult stones were treated. Our present incidence of auxiliary procedures (including repeated lithotripsy) of $11.4 \%$ and increased number of planned combination treatments confirms that prediction.

In our earlier reports we concluded that lithotripsy was a specialised urological procedure, and patients in whom it was carried out must be managed at a "stone centre" where advanced endourological skill was available. This report on 1000 cases reaffirms that and reiterates the experience of others that extracorporeal shockwave lithotripsy is a safe, effective, and economical method of treating renal and ureteric calculi with an exceptionally low morbidity. We emphasise that in these 1000 cases an overall success rate of $91.8 \%$ was achieved $(96.3 \%$ with $1 \mathrm{~cm}$ stones) with no mortality, minimal morbidity, and short hospital stay. Open nephrolithotomy is still indicated for patients who have large staghorn calculi. For most patients, however, modern technology has made open surgery for calculous disease obsolete.

\section{References}

1 Webb DR, McNicholas TA, Whitfield HN, Wickham JEA. Extra corporeal shock wave lithotripsy, endourology and open surgery: the management and follow-up of 200 patients with lithotripsy, endourology and open surgery: the manag
urinary calculi. Ann R Coll Surg Engl 1985;67:337-40.

2 Coptcoat MJ, Webb DR, Kellett MJ, et al. The complications of extra corporeal shock wave lithotripsy: management and prevention. Br $\mathcal{f}$ Urol 1986;58:578-80.

3 Eisenberger F, Fuchs G, Miller K, Bub P, Rassweiler J. Extra corporeal shock wave lithotripsy (ESWL) and endourology: an ideal combination for the treatment of kidney stones. World foumal of Urology 1985;3:41-7.

4 Fuchs G, Miller K, Rassweiler J, Eisenberger F. Extra corporeal shock wave lithotripsy: one year experience with the Dornier lithotripter. Eur Urol 1985;11:145-9.

5 Lingeman JE, Newman D, Mertz JHO, et al. Extracorporeal shockwave lithotripsy, the Methodist Hospital of Indiana experience. F Urol 1986;135:1134-7.

6 Charig CR, Webb DR, Payne SR, Wickham JEA. Comparison of treatment of renal calculi by open surgery, percutaneous nephrolithotomy, and extracorporeal shockwave lithotripsy. $\mathrm{Br}$ Med f 1986;292:879-82.

7 Jocham D, Chaussy C, Schmiedt E. Extra corporeal shock wave lithotripsy. Urol Int 1986;41:357-68.

8 Drach GW, Dretler S, Fair W, et al. Report of the United States cooperative study of extra corporeal shock wave lithotripsy. $f$ Urol 1986;135:1127-33.

9 Palfrey ELH, Bultitude MI, Challah S, Pemberton J, Shurtleworth KED. Report on the first 1000 patients treated at St Thomas's Hospital by extra corporeal shock wave lithotripsy. Br.f Urol 1986;58:573-7.

10 Ramsay JWA, Payne SR, Gosling PT, Whitfield HN, Wickham JEA, Levison DA. The effects of Double J stenting on unobstructed ureters. Br $\mathcal{J}$ Urol 1985;57:630-4.

11 Wickham JEA, Webb DR, Payne SR, Kellet MJ, Watkinson G, Whitfield HN. Extracorporeal shock wave lithotripsy: the first 50 patients treated in Britain. BrMed f 1985;290:1188-9.

(Accepted 29. June 1987)
Is microscopic haematuria a common condition in women over 60 and what advice should a patient be given with this condition?

Does the patient really have microscopic haematuria? Before further steps are contemplated another specimen should be collected carefully and a sterile sample sent for culture. Dipstick tests for blood in urine are very sensitive, although there are occasionally causes for false positive results. The prevalence of microscopic haematuria in fit and healthy women over 60 years old is not known. A recent study of 10050 men undergoing BUPA "health screening" showed a prevalence of occult haematuria of $2.5 \%$."

If there is persistent microscopic haematuria the obvious concern is that there may be a urological neoplasm. Hence referral is instinctively to urologists. If proteinuria ( $1+$ or more) on stick testing is present, however, a glomerular source of bleeding is more likely and referral to a nephrologist may be more appropriate. Phase contrast microscopy should, in theory, differentiate between the dysmorphic red cells found with glomerular disease and the symmetrical red cells found with urological disease. The presence of the former should prevent unnecessary tests, but this simple investigation is probably not yet widely performed and accepted in the United Kingdom.

Any 60 year old woman sent to a urologist with persistent microscopic haematuria will have her urine cultured and sent for cytological examination on two or three occasions and will have intravenous pyelography and cystoscopy. In several studies of patients with microscopic haematuria (referred to urologists) about $20 \%$ consistently have appreciable pathology, half of whom have a neoplasm. ${ }^{13}$ Of the rest, $50 \%$ have no cause found and $30 \%$ have some trivial pathology. Furthermore, men are four times more likely to have a neoplasm than women.

There must be a high probability that if haematuria is found in a 60 year old there is some relevant symptom for the test to be done in the first place. Therefore in urological reports of persistent microscopic haematuria there is probably a higher percentage of pathology compared with that in a population in which the haematuria was discovered at a routine examination. In the BUPA study two out of 76 patients who were followed up had a neoplasm. ${ }^{2}$ The patient should be advised to have further investigations. But there is probably a less than $5 \%$ chance of a neoplasm being found if the haematuria was an incidental finding.-G NEILD, senior lecturer in nephrology, London.

\footnotetext{
Woodhouse CRJ. Symptomless abnormalities: microscopic haematuria. Br $\mathcal{J}$ Hosp Med 1982;27: 163-8.

Ritchie CD, Bevan EA, Collier SJ. Importance of occult haematuria found at screening. Br Med f 1986;292:681-3.

3 Golin AL, Howard RS. Asymptomatic microscopic haematuria. $f$ Urol 1980;124:389-91.
} 